\title{
Das erste Jahr
}

\section{Ursina Pally Hofmann}

Dr. iur., Generalsekretärin der FMH

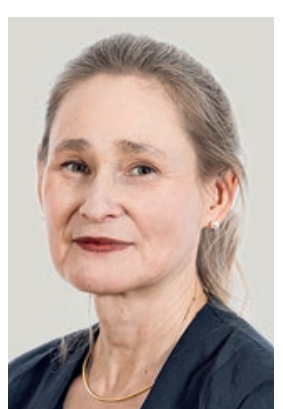

Sie werden wissen, was ich mit diesem Titel anspreche. Wir werden bald das erste Jahr mit dem unwillkommenen kleinen Gast, der sich erfolgreich in uns vermehrt und unter uns verbreitet, hinter uns haben.

Allenfalls erinnern Sie sich noch an mein Editorial vor einem Jahr betreffend Chancen und Risiken im Allgemeinen. Danach verwirklichen sich Risiken ungefragt. Wer hätte damals gedacht, dass uns allen so bald ein derart unerwünschtes Geschenk zugetragen würde? Nicht, dass wir es nicht für möglich erachtet hätten oder nicht wenigstens teilweise darauf vorbereitet gewesen wären. Wenn sich das Risiko dann aber verwirklicht, merkt man sehr schnell, wie wenig man darauf vorbereitet ist, weil vieles anders ist als gedacht. Es hat uns mehr oder weniger eiskalt erwischt.

Und das unter - im Vergleich zu anderen Regionen der Welt - fast schon perfekten Bedingungen. Dennoch fühlt es sich nicht gut an, und auch wir sind schwer getroffen worden.

Was machen wir damit in persönlicher und gesellschaftlicher Hinsicht? Ist sich seit dieser Pandemie jede und jeder ständig bewusst, wie fragil unser Gleichgewicht hinsichtlich vieler Aspekte des menschlichen Daseins ist? Wie wenig es braucht, um uns aus der Bahn zu werfen, wie hilflos wir uns fühlen können? Oder denken wir vielmehr, dass alles so weitergehen wird wie bis anhin, sobald der ungebetene Gast nicht mehr da ist oder wir ihn gezähmt haben? Ein verlockender Gedanke, der aber kaum gerechtfertigt sein dürfte.

Bald werden wir das erste Jahr mit dem unwillkommenen kleinen Gast hinter uns haben.

Haben wir etwas daraus gelernt? Für uns persönlich vielleicht, weil uns bewusst wurde, welche Einschränkungen und Ängste am meisten schmerzen, oder wir in unserer diesbezüglichen Selbstwahrnehmung bestätigt worden sind.

Zu hoffen ist, dass wir daraus nicht nur für uns persönlich etwas lernen können. Das Bewusstsein für Risiken, die uns im Falle ihrer Verwirklichung plötzlich und hart treffen, wie auch immer sie geartet sein mögen, sollte eigentlich gewachsen sein. Damit meine ich nicht nur das Bewusstsein, eine nächste Pandemie könne wieder auftreten. Es gibt Risiken, die ganz anders gelagert sind, aber einen mindestens ebenso grossen Schaden anrichten, wenn sie da sind. Was würde wohl passieren, wenn unsere virtuelle Welt, die wir in diesen letzten Monaten ausgebaut haben, nicht mehr existierte? Unter anderem darauf haben wir uns möglichst rasch und umfassend vorzubereiten. Dieses Jahr konnten wir alle am eigenen Leib erfahren, dass Risiken kein theoretisches Konstrukt irgendwelcher Pessimisten sind.

\section{Es wird nicht das letzte Jahr mit diesem ungebetenen Gast sein, aber wir können das Beste daraus machen.}

Diese Krise birgt auch Chancen. Wir Mitarbeitende des Generalsekretariats der FMH haben aufgrund der jüngsten Entwicklung innert kürzester Zeit eine virtuelle Ärztekammersitzung organisieren müssen, welche komplexe rechtliche und technische Anforderungen zu erfüllen hatte. Das geht nur mit Fachkompetenz, Flexibilität, gegenseitigem Respekt und Vertrauen. Wie erfreulich, dass uns das gelungen ist und ich solch ein überzeugendes Team leiten darf.

Ich bin überzeugt: Viele unter Ihnen werden Ihre Chance ebenfalls genutzt haben oder dies noch tun können.

Es wird nicht das letzte Jahr mit diesem ungebetenen Gast sein. Und es wird nicht das letzte unwillkommene Geschenk sein, das wir so schnell nicht wieder loswerden. Wir können aber das Beste daraus machen, nicht aufgeben, nach Lösungen suchen und umgehend damit beginnen, uns besser vorzubereiten. In der Hoffnung, dass wir die jeweiligen Herausforderungen meistern und irgendwann wieder - vermeintlich Herr der Lage sein werden. L'espoir fait vivre. 